\title{
miR-129-2 suppresses proliferation and migration of esophageal carcinoma cells through downregulation of SOX4 expression
}

\author{
MIN KANG ${ }^{1}$, YUMEI LI ${ }^{2}$, WENQI LIU ${ }^{1}$, RENSHENG WANG ${ }^{1}$, ANZHOU TANG $^{1}$, \\ $\mathrm{HONG} \mathrm{HAO}^{3}$, ZHENGUO $\mathrm{LIU}^{3}$ and HESHENG OU ${ }^{2}$ \\ ${ }^{1}$ The First Affiliated Hospital, ${ }^{2}$ College of Pharmacy, Guangxi Medical University, Nanning, Guangxi, P.R. China; \\ ${ }^{3}$ Davis Heart and Lung Research Institute and Division of Cardiovascular Medicine, \\ The Ohio State University Medical Center, Columbus, OH, USA
}

Received December 11, 2012; Accepted April 11, 2013

DOI: $10.3892 /$ ijmm.2013.1384

\begin{abstract}
We report the emerging role of microRNA (miRNA) deregulation associated with activation of an oncogene SOX4 (a member of the SRY-related HMG-box) in esophageal carcinoma. Paired esophageal cancer and adjacent non-tumor tissues were obtained from 42 patients who underwent primary surgical resection for esophageal cancer. Experiments such as real-time PCR, western blot analysis, luciferase-reporter assay, cell proliferation and colony formation assays, in vitro migration and invasion assays, and a wound-healing assay were performed to determine the effects of miR-129-2. We found that SOX4 expression was elevated $(\mathrm{P}<0.005)$ in esophageal tumors $(n=42)$ when compared with its expression in the controls $(n=42)$. Compared with the normal esophageal tissues, the expression of miR-129-2 was downregulated in 27 of 31 primary esophageal tumors, while the expression of SOX4 was upregulated $(\mathrm{P}<0.001)$. Restoration of miR-129-2 by transfection with an miRNA expression plasmid led to a decrease in SOX4 expression, which was accompanied by reduced migration and proliferation of the cancer cells. These results suggest that aberrant expression of SOX4 is associated with repression of miR-129-2, and restoration of miR-129-2 suppresses the migration and proliferation of esophageal cancer cells. Our results demonstrated that the deregulation of miR-129-2 leads to aberrant SOX4 expression, presenting a new paradigm in which the restoration of miRNA suppresses its oncogenic target in esophageal cancer.
\end{abstract}

Correspondence to: Dr Hesheng Ou, College of Pharmacy, Guangxi Medical University, 22 Shuangyong Road, Nanning, Guangxi 530021, P.R. China

E-mail: hsou01@126.com

Dr Zhenguo Liu, Davis Heart and Lung Research Institute and Division of Cardiovascular Medicine, The Ohio State University Medical Center, DHLRI Suite 200, 473 West 12th Ave., Columbus, $\mathrm{OH} 43210$, USA

E-mail: zhenguo.liu@osumc.edu

Key words: SOX4, miR-129-2, esophageal cancer, proliferation, migration

\section{Introduction}

Esophageal cancer is the eighth most common cancer and the sixth common cause of cancer-related death in the world (1). Esophageal cancer includes two subtypes, esophageal squamous cell carcinoma (ESCC) and esophageal adenocarcinoma (EAC). The distribution of these subtypes is different. For example, ESCC is the most frequent subtype of esophageal cancer in China, and EAC is the major form of esophageal cancer in the USA. Both types are generally diagnosed at a late stage and are associated with a poor prognosis, with a 5 -year survival of $<10 \%$. Currently, clinical treatment for this tumor includes chemotherapy, radiation therapy, and esophagogastric resection. However, an extremely poor survival is imminent for many patients (particularly with EAC) despite such treatment, suggesting that such tumors are resistant to standard therapy. Therefore, more effective treatment strategies are needed in order to reduce the morbidity and mortality associated with such tumors, particularly treatments targeting cancer invasion and metastasis. Yet, the molecular mechanism(s) involved in the invasion and metastasis of such tumors is poorly understood.

SOX4 is a member of the SRY-related HMG-box (SOX) transcription factor family and is involved in a variety of human malignancies, including prostate, hepatocellular, and lung cancers, with poor prognostic features and advanced disease status (2-5). The SOX4 gene encodes a protein of 474 amino acids with three distinguishable domains, including an HMG box, a glycine-rich region, and a serine-rich region. The HMG box serves as a DNA-binding region, whereas the serine-rich domain serves as a transactivation domain (6). The central domain containing the glycine-rich region located between the HMG box and serine-rich domains serves as a novel functional region for promoting apoptotic cell death (7). In both knock-in and knock-out cells, SOX4 has showed its oncogenic potentials due to aberrant transformation and proliferation and metastatic capability $(3,8)$. Recently, it was found that transcriptional targets of SOX4 are associated with tumor metastasis and microRNA (miRNA) processing $(6,8)$. However, few reports have provided direct evidence indicating a correlation between aberrant SOX4 expression and miRNA alterations in tumorigenesis. 
miRNAs, a class of small non-coding RNAs, are known to regulate target gene expression by mRNA degradation or translational inhibition through imperfect paring at the 3'-end of untranslated regions (UTRs) (9). Increasing evidence suggests that abnormal miRNA expression may be closely associated with epigenetic perturbations in cancer cells (10). Specifically, several tumor-specific genes have been identified as targets of miRNAs in cancer $(8,11,12)$, indicating that miRNAs may play a pivotal role in tumorigenesis and may serve as novel targets for cancer therapy.

In this study, we report that SOX4 is overexpressed in esophageal cancer. The expression of miR-129-2, which is computationally predicted as an upstream regulator of $S O X 4$, was correlated with $S O X 4$ levels in esophageal cancer samples. In esophageal cancer cell lines, we further revealed that restoration of miR-129-2 by transfection with an miRNA expression plasmid led to decreased SOX4 expression, and coincided with reduced migration and proliferation of cancer cells.

\section{Materials and methods}

Patients and tissue samples. The use of human tissues in this study was approved by the Human Research Ethics Committee of Guangxi Medical University. Paired esophageal cancer and adjacent non-tumor tissues were obtained from 42 patients who underwent primary surgical resection for esophageal cancer with informed consent between March 2011 and April 2012 at The First Affiliated Hospital, Guangxi Medical University in China. The clinical stage was determined according to the revised International Staging System. The tumor or nontumor tissues were verified by pathological examination. The pathological stage, grade, and lymph nodal status were assessed independently by three experienced pathologists. The clinical characteristics of the patients, including age, gender, pathology, as well as tumor-node-metastasis (TNM) staging, were collected and assessed.

Cell culture and transfection. The NMC109 cell line was cultured in RPMI-1640 medium containing $10 \%$ fetal bovine serum (FBS), $100 \mathrm{IU} / \mathrm{ml}$ penicillin and $100 \mu \mathrm{g} / \mathrm{ml}$ streptomycin in humidified $5 \% \mathrm{CO}_{2}$ at $37^{\circ} \mathrm{C}$. The $\mathrm{NMC} 109$ cell line was obtained from the Shanghai Cell Bank, Chinese Academy of Sciences.

The miR-129-2 mimics and miR-129-2 inhibitor were synthesized by RiboBio Co., Ltd. (Guangzhou, China). For transfection, cells were grown to $90 \%$ confluence, and transfected with miR-129-2 mimics or its inhibitor with Lipofectamine 2000 by incubation with Opti-Mem I media for $4 \mathrm{~h}$. The cells were then transferred into fresh RPMI-1640 with $10 \%$ FBS. After incubation for $24 \mathrm{~h}$, the culture medium was replaced, and fluorescent images were utilized to monitor transfection efficiency. After $48 \mathrm{~h}$, cells were harvested for analysis. All assay conditions were performed in triplicate.

Immunohistochemistry. The selected tumor tissues and tissues adjacent to the tumor (TAT, collected at a distance of $5 \mathrm{~cm}$ from the tumor) were used to constructed TMA slides. Paraffin sections were cut and mounted on glass slides, and 5- $\mu \mathrm{m}$ sections from formalin-fixed and paraffin-embedded specimens were deparaffinized using xylene and rehydrated in graded ethanol.
Samples were then pre-incubated with $3 \% \mathrm{H}_{2} \mathrm{O}_{2}$ to eliminate endogenous peroxidase activity. Antigen retrieval was achieved by heating the sections (for $2 \mathrm{~min}$ to $100^{\circ} \mathrm{C}$ ) in citric acid buffer (0.01 mol/1, pH 6.0). Immunohistochemistry was performed using a 2-step method. Briefly, sections were incubated overnight at $37^{\circ} \mathrm{C}$ with the primary antibody. The components of the Envision detection system were applied with an anti-mouse polymer (EnVision1/HRP/Mo; Dako, Glostrup, Denmark). The SOX4 primary antibodies were mouse monoclonal (American Research Products, Belmont, MA, USA), and diluted in a ratio of 1:200 prior to use. Negative controls were carried out using the same procedures but without the primary antibody.

The percentage of positive tumor cells was determined by three observers, and the average of 3 scores was calculated. For scoring, the following categories were defined: none, 0; mild, 1; moderate, 2; strong, 3 for intensity of staining; $<5 \%$, $0 ; 5-25 \%, 1 ;>25-50 \%, 2 ;>50 \%, 3$ for the percentage of positive staining. A general score combining both intensity and percentage staining was scored as follows: $0-1$, negative (-); 2-4, moderate $(+)$; 5-6, strong $(++)(13,14)$.

Real-time PCR. Total RNA was extracted using TRIzol reagent (Invitrogen, USA) for both miR-129-2 and SOX4 mRNA analyses. For detection of miR-129-2 expression, stem-loop RT-PCR was performed using SYBR Premix Ex Taq ${ }^{\mathrm{TM}}$ (Takara) according to the manufacturer's protocol. Relative expression was evaluated by comparative CT method and normalized to the expression of U6 small RNA. Primers for miR-129-2 were: stem-loop RT primer, 5'-CAGAACAGTGTCGTGACAGTG ACGATATTGTTCTGGCAAGC-3'; forward, 5'-GCGACTGA CGTCTTTTTGCGGTCTGG-3' and reverse primer, 5'- CAGA ACAGTGTCGTGACAGTGACGAT-3'. Primers for U6 were: RT primer, 5'-GTCGTATCCAGTGCAGGGTCCGAGGTA TTCGCACTGGATACGACAAAAATATG-3'; forward, 5'-GC GCGTCGTGAAGCGTTC-3' and reverse primer, 5'-GTGCA GGGTCCGAGGT-3'. For detection of SOX4 mRNA expression, real-time-PCR was performed using QuantiTect SYBR-Green PCR kit (Qiagen, USA). GAPDH was used to normalize SOX4 mRNA expression levels. Forward and reverse primer sequences for SOX4 mRNA were as follows: SOX4 forward, 5'-GTGAGCGAGATGATCTCGGG-3' and reverse, 5'-CAGGTTGGAGATGCTGGACTC-3'; GAPDH forward, 5'-AACTTTGGCATTGTGGAAGG-3' and reverse, 5'-ACACA TTGGGGGTAGGAACA-3' (15). All the experiments were performed in triplicate. The expression of SOX4 and miR-129-2 were normalized to GAPDH and $\mathrm{U} 6$, respectively, and were obtained by: $2^{-\Delta \mathrm{Ct}} . \Delta \mathrm{C}_{\mathrm{t}}$ was calculated as $\mathrm{C}_{\mathrm{t}}(S O X 4)-\mathrm{C}_{\mathrm{t}}$ (GAPDH) or $\mathrm{C}_{\mathrm{t}}$ (miR-129-2) - $\mathrm{C}_{\mathrm{t}}$ (U6).

Western blot analysis. Proteins $(30 \mu \mathrm{g})$ were separated on $8 \%$ SDS-PAGE and transferred to nitrocellulose membranes (Bio-Rad, Hercules, CA, USA). The membranes were blocked with 5\% non-fat milk and incubated with mouse anti-SOX4 $(1: 1,000 ;$ Abcam, Southampton, UK) or mouse anti-actin (1:5,000; Sigma) in 5\% non-fat milk in TBST at room temperature for $2 \mathrm{~h}$, followed by exposure to a goat anti-rabbit or anti-mouse secondary antibody conjugated with horseradish peroxidase. Signals of the immunoreactive bands were visualized using the ECL detection system (Pierce Biotechnology, Inc., Rockford, IL, USA). 
Luciferase-reporter assay. The human DNA fragment containing the 3'-UTR segments of SOX4 mRNA containing the miR-129-2 binding sites were PCR amplified and cloned into the Xba1 site of the pGL3 vector (Promega, Madison, WI, USA) (referred to as pGL3-SOX4-wt). With pGL3-SOX4wt as a template, mutations in the miR-129-2 binding sites were performed using a QuikChange ${ }^{\circledR}$ Site-Directed Mutagenesis kit (Stratagene, La Jolla, CA, USA) (referred to as pGL3-SOX4-mut). NMC109 cells were transfected in 24-well plates with the wild-type or mutant reporter plasmid using Lipofectamine 2000. After transfection for $6 \mathrm{~h}$, cells were transfected again with miR-129-2 or negative control. Luciferase activity was measured using the dual luciferase assay system (Promega) after a 36-h incubation.

Cell proliferation and colony formation assays. Cells were plated in 96-well plates (5,000 cells/well), incubated for $48 \mathrm{~h}$, and then transfected with $50 \mathrm{nmol} / 1$ of miR-129-2 mimics or its inhibitor or the negative control. At the end of the incubation, the cell proliferation reagent WST-8 $(10 \mu \mathrm{l})$ was added to each well and incubated for $3 \mathrm{~h}$ at $37^{\circ} \mathrm{C}$. Viable cell numbers were estimated by measurement of the optical density (OD) at $450 \mathrm{~nm}$.

For the colony formation assay, NMC109 cells were transfected with $50 \mathrm{nmol} / 1$ of miR-129-2 mimics or its inhibitor, cultured in media containing $10 \% \mathrm{FBS}$; the medium was replaced every 3 days. After incubation for 14 days, cells were fixed with methanol and stained with $0.1 \%$ crystal violet (Sigma). Visible colonies were manually counted. Triplicate wells were measured for each group.

In vitro migration and invasion assays. For the Transwell migration assay, $10 \times 10^{4}$ cells were plated in the top chamber with a non-coated membrane (24-well insert; 8 - $\mu \mathrm{m}$ pore size; BD Biosciences). For the invasion assay, $2 \times 10^{5}$ cells were plated in the top chamber with a Matrigel-coated membrane (24-well insert; $8-\mu \mathrm{m}$ pore size; BD Biosciences). In both assays, cells were plated in medium without serum, and medium supplemented with $10 \%$ serum was used as a chemoattractant in the lower chamber. The cells were incubated for 24 to $36 \mathrm{~h}$. Cells that did not migrate or invade through the pores were removed by a cotton swab. Filters were fixed with $90 \%$ ethanol, stained with $0.1 \%$ crystal violet, photographed and cell numbers were counted.

Wound-healing assay. The cultured cells were transfected with $50 \mathrm{nM}$ miR-129-2 mimics or negative control. At $24 \mathrm{~h}$ post-transfection, the cells were allowed to reach confluence before dragging a 1-ml sterile pipette tip through the monolayer. Cells were then washed and allowed to migrate for 12 or $24 \mathrm{~h}$. At time 0,12 and $24 \mathrm{~h}$ post-wounding, images were captured. Cell motility was determined according to the percentage of the repaired area (16). Each assay group was measured in triplicate.

Statistical analysis. All data are expressed as means \pm SEM of three independent experiments unless specified otherwise. Independent Student's t-test was used for comparisons between groups. One-way ANOVA was applied for multiple group comparisons with post hoc Bonferroni correction for
Table I. Characteristics of the esophageal cancer patients in this study.

\begin{tabular}{lr} 
Characteristics & No. of patients $(\%)$ \\
\hline Median age (range), in years & $48(30-74)$ \\
Gender & \\
Male & $31 / 42(73.8)$ \\
Female & $11 / 42(26.2)$ \\
Histological type & \\
Squamous & $10 / 42(23.8)$ \\
Adenocarcinoma & $32 / 42(76.2)$ \\
TNM stage & \\
I + II & $12 / 42(28.6)$ \\
III + IV & $30 / 42(71.4)$ \\
Lymph node metastasis & \\
Positive & $24 / 42(57.1)$ \\
Negative & $18 / 42(42.9)$ \\
Differentiation & \\
Well & $6 / 42(14.3)$ \\
Moderate & $11 / 42(26.2)$ \\
Poor & $25 / 42(59.5)$ \\
Tumor size (cm) & \\
$\leq 5$ & $17 / 42(40.5)$ \\
$>5$ & $25 / 42(59.5)$ \\
Distal metastasis & \\
Positive & \\
Negative & \\
\hline
\end{tabular}

TNM, tumor-node-metastasis

multiple comparisons using SPSS 13.0 for Windows (SPSS, Inc., Chicago, IL, USA). Differences were considered statistically significant at P-values $<0.05$.

\section{Results}

miR-129-2 downregulation correlates with advanced TNM stage in esophageal cancers. The clinical and pathological data of the 42 esophageal cancer patients are summarized in Table I. The expression level of miR-129-2 was evaluated in 42 paired esophageal cancer tissues and adjacent non-tumor tissues by real-time RT-PCR. miR-129-2 was downregulated in 35 tumor tissues when compared with the matched non-tumor tissues (Fig. 1A). Specifically, downregulation of miR-129-2 in esophageal cancer tissues was observed in 28 of 30 patients with stage III or IV tumors. The difference in miR-129-2 expression between the tumor and non-tumor tissues was statistically significant (Fig. 1B). Furthermore, the association between miR-129-2 and the clinicopathologic factors (Table I) was examined in tumor tissues. Our results revealed that miR-129-2 downregulation was associated with advanced clinical TNM stage (Fig. 1C), distal metastasis (Fig. 1D) and lymph node metastasis (Fig. 1E). 

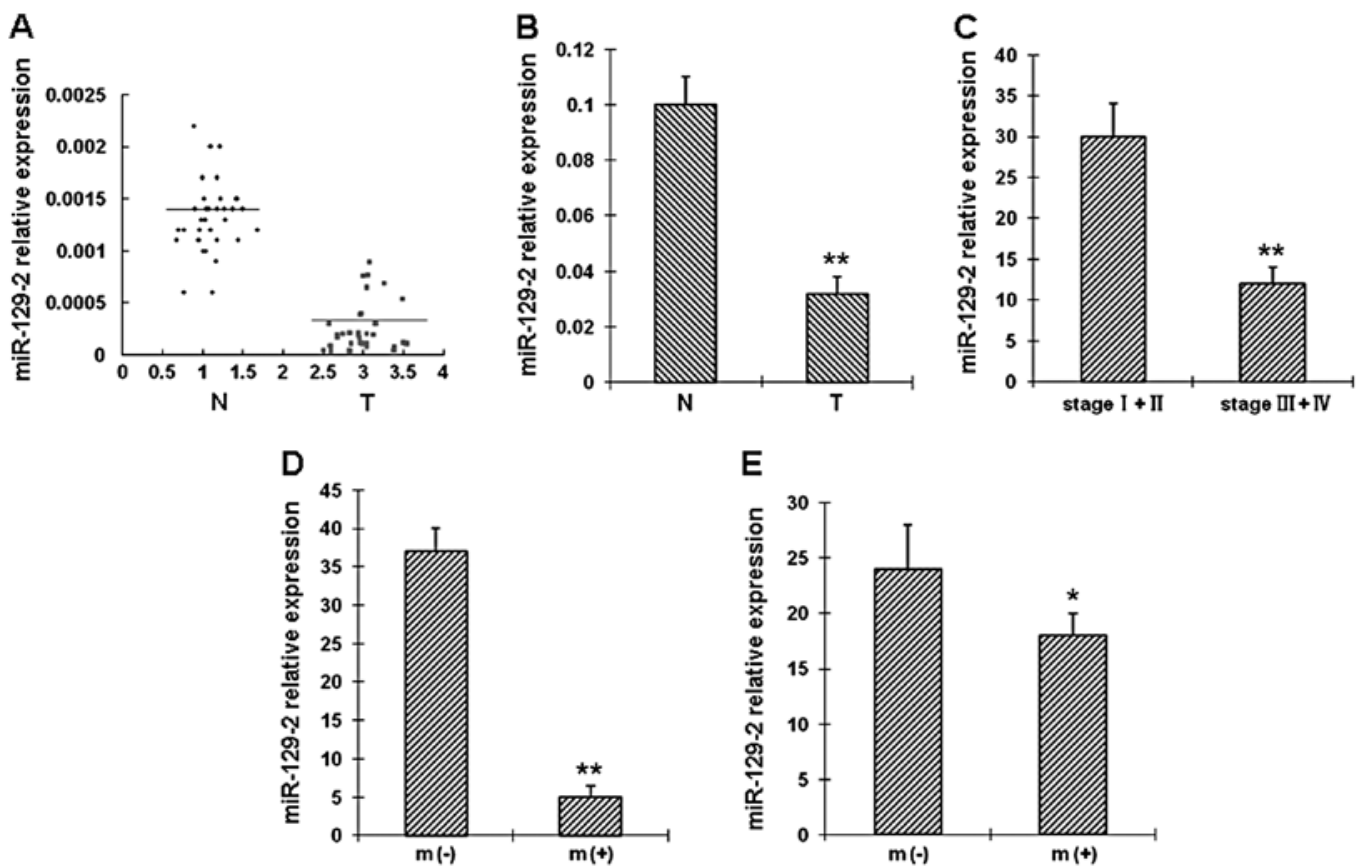

Figure 1. Downregulation of miR-129-2 in esophageal carcinoma tissues and its clinical significance. Paired esophageal cancer and adjacent non-tumor tissues were obtained from 42 patients who underwent primary surgical resection for esophageal cancer. miR-129-2 expression was analyzed by RT-PCR, and was normalized to U6 expression. (A) Relative expression of miR-129-2 in esophageal carcinoma tissues $(\mathrm{n}=42)$ in contrast with matched non-tumor tissues $(\mathrm{n}=42) . \mathrm{N}$, non-tumor tissue; $\mathrm{T}$, tumor tissue. (B) miR-129-2 expression in tumor tissues and in adjacent non-tumor tissues. $\mathrm{N}$, non-tumor tissue; $\mathrm{T}$, tumor tissue ${ }^{* *} \mathrm{P}<0.001$. (C) miR-129-2 expression in patients with stages III-IV and in those with stages I-II. ${ }^{* *} \mathrm{P}<0.001$. (D) miR-129-2 expression in patients with distal metastasis $[\mathrm{m}(+)]$ and in those without metastasis $[\mathrm{m}(-)] .{ }^{* *} \mathrm{P}<0.001$. (E) miR-129-2 expression in patients with lymph node metastasis $[\mathrm{m}(+)]$ and in those without metastasis $[\mathrm{m}(-)] .{ }^{*} \mathrm{P}<0.05$.

SOX4 protein levels are inversely correlated with miR-129-2 expression in esophageal cancer tissues. Several genes have been identified as the putative targets of miR-129-2 by computational prediction. In this study we focused on oncogene SOX4. Among the 42 pairs of matched esophageal cancer specimens, 20 pairs were randomly selected for analysis of SOX4 mRNA by RT-PCR and SOX4 protein by western blotting and immunohistochemistry. Expression of SOX4 was absent in 12 of the 20 normal esophageal samples $(60 \%)$ and in 4 of the $20(20 \%)$ carcinomas. Representative examples of SOX4 protein expression in esophageal cancer samples are shown in Fig. 2A-D. We also examined the association between SOX4 protein and miR-129-2 in these 20 esophageal tumor samples. A statistically significant inverse correlation was observed between miR-129-2 and SOX4 protein (Fig. 2E); low expression of miR-129-2 was correlated with high amounts of SOX4 protein.

SOX4 is a direct target of $m i R-129-2$. To determine whether miR-129-2 directly targets the 3'-UTRs of SOX4 mRNA, we cloned a sequence with the predicted target sites of miR-129-2 or a mutated sequence with the predicted target sites downstream of the pMIR luciferase reporter gene. When the wild-type or mutation-type vector was transfected with miR-129-2, the luciferase activity of the wild-type vector was significantly decreased $(\mathrm{P}<0.001)$ when compared with the mutation-type vector (Fig. 3). When the wild-type or mutationtype vector was transfected with the negative control miRNA, there was no significant difference between the wild-type or mutation-type vector. These data suggest that miR-129-2 may play a major role in the regulation of SOX4 expression.
miR-129-2 overexpression restrains cell growth and SOX4 expression in esophageal carcinoma cell lines. To investigate the biological function of miR-129-2 in esophageal cancer, we first elevated the expression level of miR-129-2 in NMC109 cells by transfecting the cells with $25 \mathrm{nM}$ of the miR-129-2 high-expression plasmid. Overexpression of miR-129-2 significantly suppressed the growth of NMC109 cells (Fig. 4A). Blocking endogenous miR-129-2 through transfection with $50 \mathrm{nM}$ of the inhibitor resulted in more rapid proliferation compared to the control cells. Furthermore, transfection of miR-129-2 mimics in the NMC109 cells resulted in a significant decrease in colony formation in soft agar compared with the control mimics (Fig. 4B). Conversely, silencing of miR-129-2 in NMC109 cells increased the colony formation (Fig. 4C). These results indicate that miR-129-2 inhibits esophageal carcinoma cell proliferation in vitro.

To analyze the effect of miR129-2 on SOX4 protein expression, we used cultured NMC109 cells transfected with miR129-2 mimics and inhibitor. Western blot analysis revealed that miR129-2 mimics significantly decreased SOX4 protein expression by $87.3 \%(0.127 \pm 0.083$ vs. $1.000 \pm 0.162, \mathrm{P}<0.01)$ (Fig. 4D). Conversely, the miR-129-2 inhibitor significantly increased SOX4 protein expression by $106.3 \%(2.063 \pm 0.181$ vs. $1.000 \pm 0.162, \mathrm{P}<0.01)$, compared with the control. These results indicate that miR-129-2 directly inhibits SOX4 protein expression in NMC109 cells.

miR-129-2 overexpression inhibits cell invasion and migration in esophageal carcinoma cell lines. Given that the expression of miR-129-2 is inversely correlated with metastasis of 
A

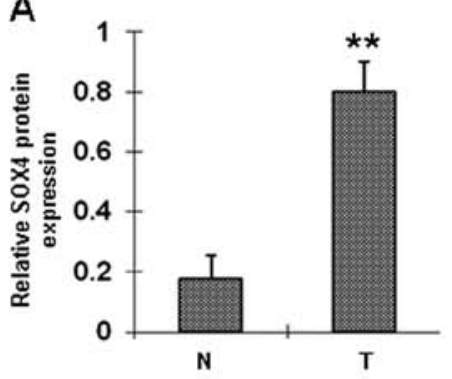

D
B
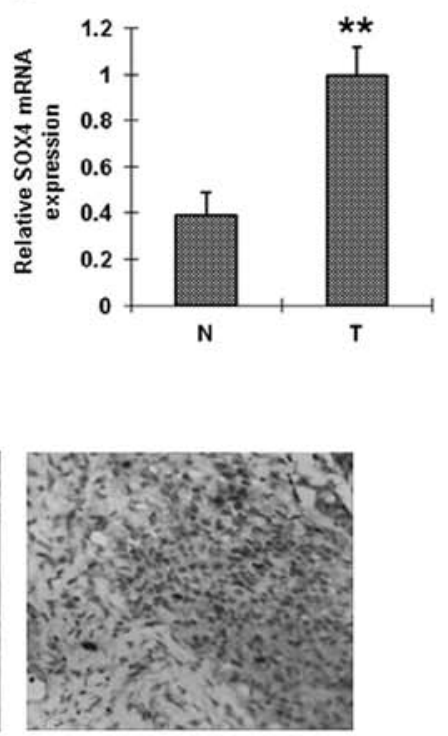

N
C
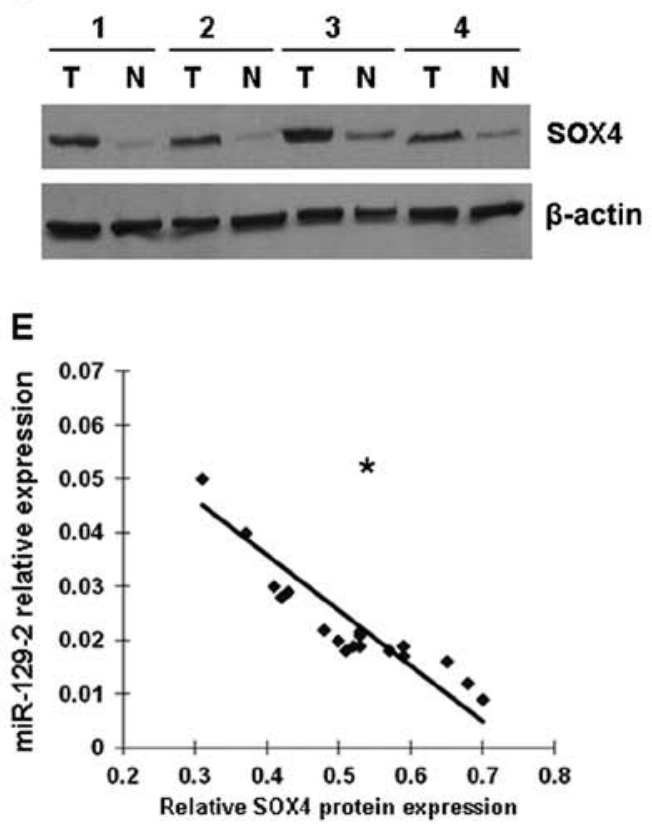

Figure 2. SOX4 protein and mRNA are upregulated in esophageal carcinoma tissues. Twenty pairs of matched esophageal carcinoma specimens were randomly selected to determine the expression levels of SOX4 mRNA and protein. (A) Relative expression of SOX4 protein in esophageal carcinoma tissues $(n=20)$ compared with matched non-tumor tissues $(n=20)$. SOX4 protein expression was detected by western blotting and normalized to $\beta$-actin protein expression. ${ }^{* *} \mathrm{P}<0.01$. N, non-tumor tissue; T, tumor tissue. (B) SOX4 mRNA levels were evaluated by qRT-PCR. The results were normalized with $\beta$-actin mRNA levels and were presented as the relative SOX4 mRNA expression ( $\left.{ }^{* * *} \mathrm{P}<0.01\right)$. (C) Representative gels for SOX4 protein levels in 4 pairs of tumor tissues (T) and matched non-tumor tissues (N). (D) Immunohistochemical staining for SOX4 with anti-SOX4 in the cancerous and normal tissues. The nuclei were counter stained with hematoxylin. N, non-tumor tissue; T, tumor tissue. (E) Correlation between miR-129-2 expression and SOX4 protein levels in esophageal carcinoma tissues (Pearson correlation $-0.752,{ }^{*} \mathrm{P}<0.05$ ).

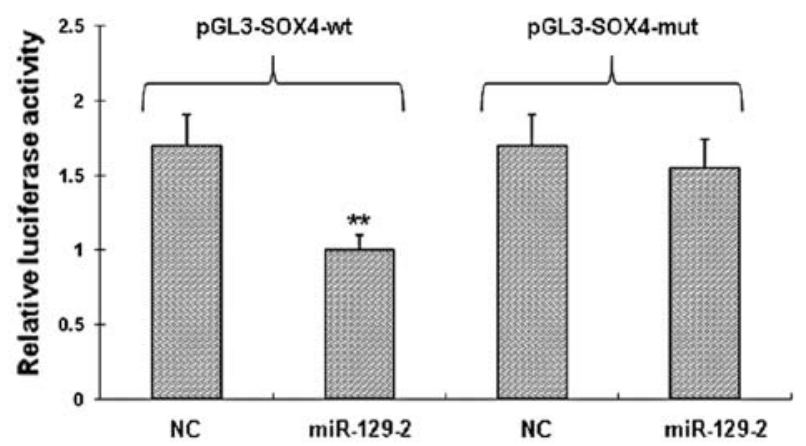

Figure 3. The 3'-UTR of SOX4 mRNA is a target for miR-129-2. The wild-type (wt) or mutant reporter plasmid was co-transfected into NMC109 cells with miR-129-2 or negative control (NC). The normalized luciferase activity in the control group was set as relative luciferase activity. Luciferase activity of pGL3-SOX4-wt was significantly decreased by miR-129-2 ( $\left.{ }^{* *} \mathrm{P}<0.01\right)$. All data are representative of three independent experiments.

esophageal cancer, we tested whether miR-129-2 affects the ability of cancer cell migration and invasion. Transwell migration and Matrigel invasion assays demonstrated that miR-129-2 significantly reduced the migration and invasion capacity of the NMC109 cells (Fig. 5A). The in vitro wound-healing assay revealed that wound repair in the NMC109 cells transfected with the miR-129-2 mimics was delayed when compared with the wound repair capacity in the cells transfected with the control mimics. miR-129-2 suppressed NMC109 cell migration by up to $69 \%(\mathrm{P}=0.013)$, compared with the control at
$24 \mathrm{~h}$ after wound scratch (Fig. 5B). These data demonstrate that miR-129-2 inhibits invasion and migration in esophageal carcinoma cell lines.

\section{Discussion}

Three major findings were revealed in this study. First, we showed that SOX4 expression was elevated in the esophageal tumor tissues and SOX4 is a direct target of miR-129-2 by virtue of its matched sequence in the 3'-UTR. Second, using RT-PCR analysis, we showed that miR-129-2 was downregulated and associated with advanced clinical TNM stage, lymph node metastasis and distal metastasis. Moreover, miR-129-2 expression had an inverse correlation with SOX4 protein levels in the esophageal cancer tissues. Finally, we showed that miR-129-2 overexpression restrained cell growth and inhibited cell invasion and migration in the cultured esophageal carcinoma cell lines.

SOX4 belongs to the group C of SOX transcription factors, which was discovered more than 15 years ago (17). Yet, the molecular properties and functions remain incompletely understood. It has been well known that SOX4 binds to the 7-bp DNA-motif AACAAAG, and transcriptionally activates its target genes $(17,18)$. A motif (AACAATA) in the human $C D 2$ gene has been recognized as the alternative motif of SOX4-binding and has been observed in vitro (19). To date, knowledge of putative complex partners and genes under control of SOX4 remains unclear. Recently, evidence indicates that SOX4 may critically control cell fate and differentiation 
A

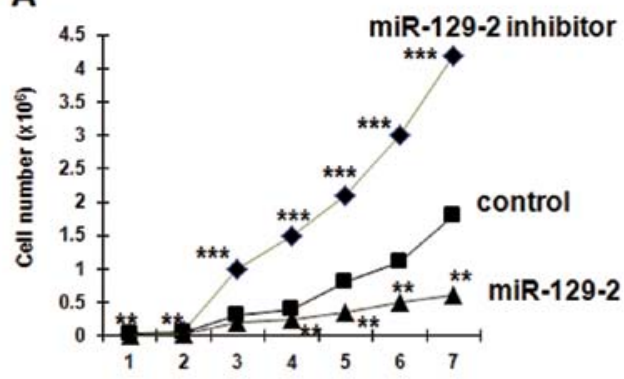

C

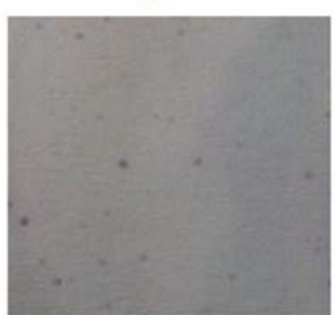

miR-129-2 inhibitor

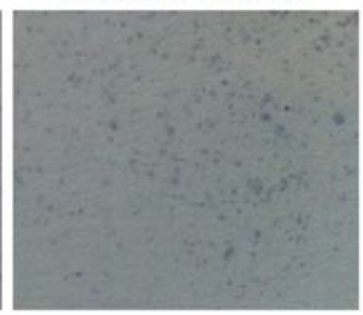

B
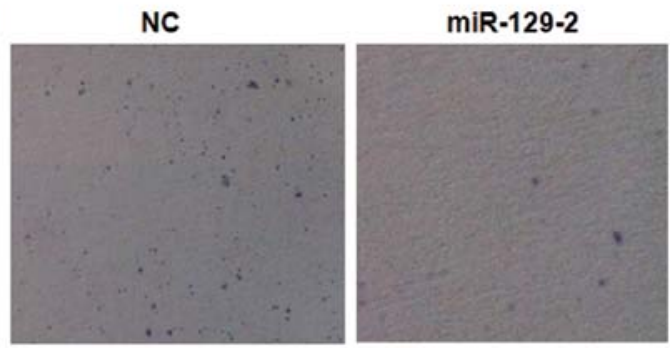

D

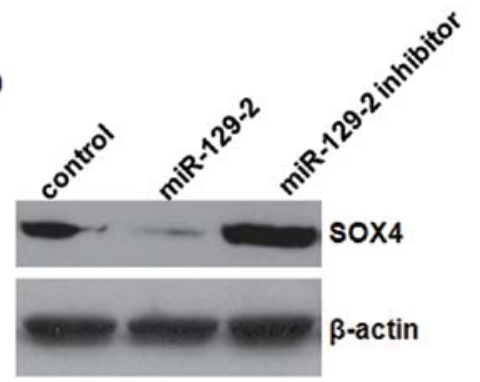

Figure 4. miR-129-2 overexpression restrains NMC109 cell growth and SOX4 expression in vitro. (A) Proliferation curve of NMC109 cells stably transfected with the empty vector (control) and the vector expressing the miR-129-2 or miR-129-2 inhibitor. (B) Colony formation of NMC109 cells transfected with miR-129-2 mimics and control mimics. (C) Colony formation of NMC109 cells with silencing of miR-129-2. (D) SOX4 protein expression in NMC109 cells transfected with miR129-2 mimics, miR-129-2 inhibitor and control as detected by western blotting.

A

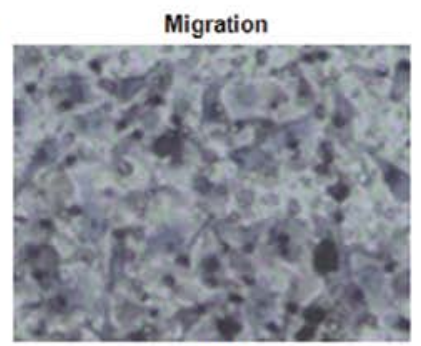

miR-NC

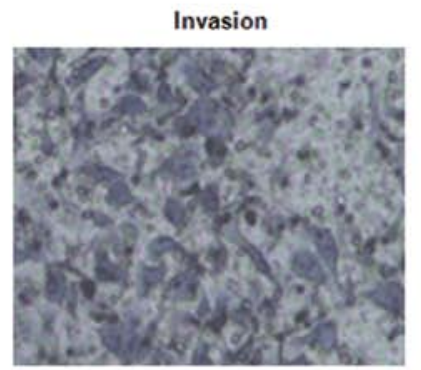

miR-NC

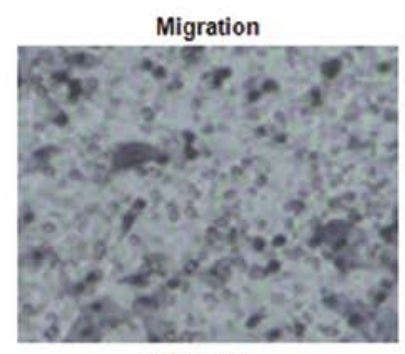

miR-129-2

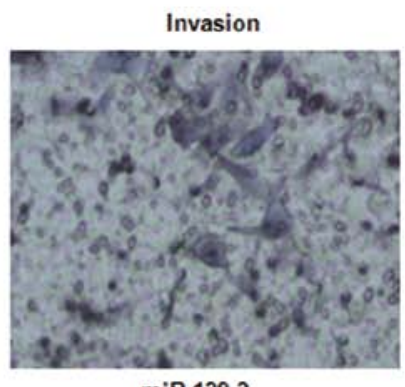

miR-129-2

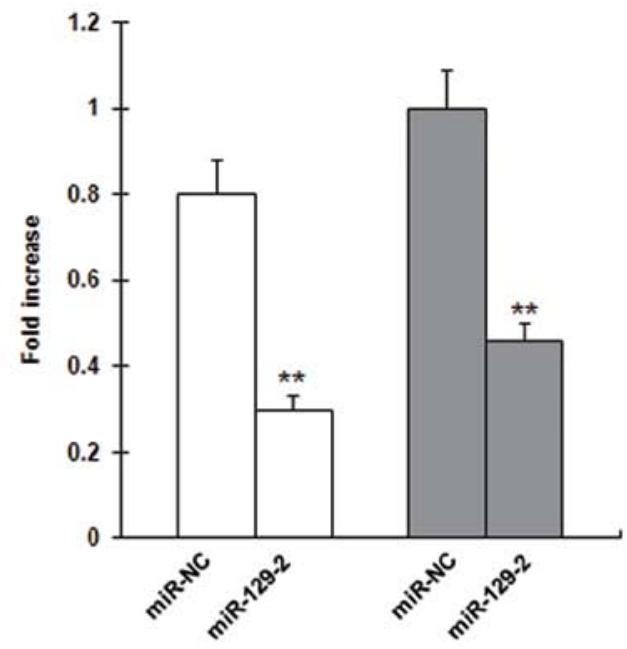

B

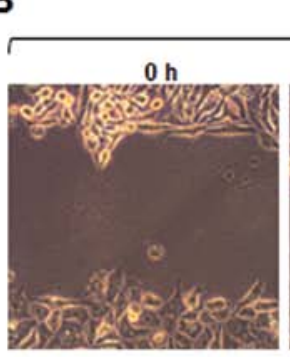

miR-NC
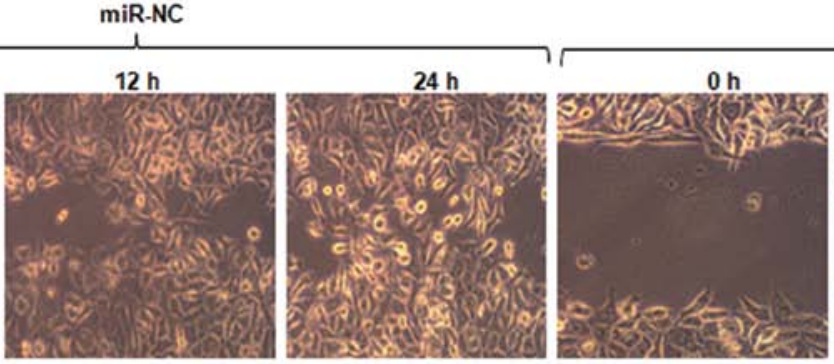

miR-129-2

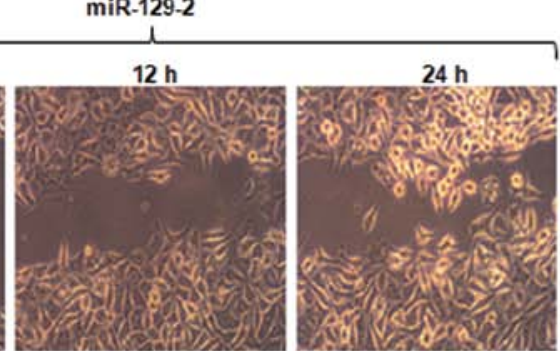

Figure 5. miR-129-2 overexpression inhibits cell invasion and migration in esophageal carcinoma cell lines. (A) Transwell migration and invasion assays of NMC109 cells transfected with miR-129-2 mimics and control mimics. (B) Representative images of NMC109 cells transfected with miR-129-2 mimics and control mimics in the wound-healing assay in vitro at 0,12 and $24 \mathrm{~h}$ after the wound scratch. Data are shown as means $\pm \mathrm{SD}(\mathrm{n}=3) .{ }^{*} \mathrm{P}<0.05 ;{ }^{* *} \mathrm{P}<0.01$. 
in major developmental processes, and that its upregulation may be a critical determinant of cancer progression (20-22). For example, SOX4b involvement in cell differentiation was suggested by its upregulation in mind bomb mutant embryos displaying accelerated pancreatic cell differentiation (23). Knockdown of SOX4 protein was found to result in reduction in cell viability and increase in apoptosis in ACC3 cells. A pro-apoptosis molecule, P53, may be responsible for induction of apoptosis, as SOX4 interacts with and stabilizes p53 protein by blocking Mdm2-mediated p53 ubiquitination and degradation (24). However, several reports found contradicting findings and showed that SOX4 expression in cancer cells could effectively drive cells into apoptosis $(25,26)$. More recently, an intriguing report showed that SOX4 positively regulated expression of known epithelial-mesenchymal transition inducers, and activated the TGF- $\beta$ pathway to contribute to epithelial-mesenchymal transition in human breast cancer (21), suggesting that SOX4 may play an important role in breast cancer progression. Overexpression of SOX4 was associated with a high incidence of myeloid leukemias and B- and T-cell lymphomas $(27,28)$.

Several reports have shown that upregulation of SOX4 occurs in a variety of human cancers, including hepatic (29), breast (30), brain (31), lung (32) and salivary gland cancers (33). However, there is no report indicating the alteration of SOX4 expression in esophageal cancer. In the present study, SOX4 was found to be upregulated in esophageal cancer tissues. This result corroborated the SOX4 alteration noted in most human cancer tissues mentioned above. Furthermore, we found that increased SOX4 protein expression was inversely associated with the downregulation of miR-129-2. To the best of our knowledge, this is the first report in esophageal tumors.

In the past decade, miRNAs have emerged as important players involved in carcinogenesis (34-36). Recently, aberrant expression of miR-129-2 has been found in different types of human cancers, including endometrial cancer (11), retinoblastoma (37), gastric cancer $(38,39)$ and colorectal cancer $(40)$. In the present study, we found that miR-129-2 expression was significantly downregulated in esophageal cancer tissues. miR-129-2 appears to be a tumor repressor which is inversely associated with its specific target gene, SOX4. This notion was further verified by results of experiments in cell culture, which revealed that overexpression of miR-129-2 inhibited cell growth and invasion. Data from recent reports also support this speculation $(38,40)$. Although evidence indicates that miR-129-2 expression may be regulated by miRNAspecific hypermethylation and histone-deacetylation $(12,38)$, the precise mechanisms involved in the downregulation of miR-129-2 in esophageal cancer tissue need to be further investigated, such as whether other transcription factor(s) take part in the role of miR-129-2 in the regulation its target gene(s).

In summary, the data presented here are consistent with the hypothesis that miR-129-2 suppresses the proliferation and migration of esophageal carcinoma through downregulation of it specific target gene SOX4. However, we emphasize that miR-129-2 restoration may be capable of controlling tumor-specific gene(s), consequently favoring cell growth and migration. Further studies should be directed toward a more complete understanding of the precise molecular mechanism(s) underlying the miRNA downregulation during tumorigenesis.

\section{Acknowledgements}

The present study was supported in part by research grant(s) from the National Natural Science Foundation of China (project nos. 30670834 and 30871186) and the Research Foundation of the Education Department of Hunan Province, China (project no. 06A060).

\section{References}

1. Dawsey SP, Tonui S, Parker RK, Fitzwater JW, Dawsey SM, White RE and Abnet CC: Esophageal cancer in young people: a case series of 109 cases and review of the literature. PLoS One 5: e14080, 2010.

2. Pramoonjago P, Baras AS and Moskaluk CA: Knockdown of Sox 4 expression by RNAi induces apoptosis in ACC3 cells. Oncogene 25: 5626-5639, 2006.

3. Medina PP, Castillo SD, Blanco S, et al: The SRY-HMG box gene, $S O X 4$, is a target of gene amplification at chromosome $6 \mathrm{p}$ in lung cancer. Hum Mol Genet 18: 1343-1352, 2009.

4. Liao YL, Sun YM, Chau GY, et al: Identification of SOX4 target genes using phylogenetic footprinting based prediction from expression microarrays suggests that overexpression of SOX4 potentiates metastasis in hepatocellular carcinoma. Oncogene 27: 5578-5589, 2008

5. Liu P, Ramachandran S, Ali Seyed M, et al: Sex-determining region $\mathrm{Y}$ box 4 is a transforming oncogene in human prostate cancer cells. Cancer Res 66: 4011-4019, 2006.

6. Scharer CD, McCabe CD, Ali-Seyed M, Berger MF, Bulyk ML and Moreno CS: Genome-wide promoter analysis of the SOX4 transcriptional network in prostate cancer cells. Cancer Res 69: 709-717, 2009

7. Dy P, Penzo-Méndez A, Wang H, Pedraza CE, Macklin WB and Lefebvre V: The three SoxC proteins - Sox4, Sox11 and Sox12 - exhibit overlapping expression patterns and molecular properties. Nucleic Acids Res 36: 3101-3117, 2008.

8. Tavazoie SF, Alarcon C, Oskarsson T, et al: Endogenous human microRNAs that suppress breast cancer metastasis. Nature 451: 147-152, 2008.

9. Bartel DP: MicroRNAs: genomics, biogenesis, mechanism, and function. Cell 116: 281-297, 2004.

10. Saito Y, Liang G, Egger G, et al: Specific activation of microRNA127 with downregulation of the proto-oncogene BCL6 by chromatin-modifying drugs in human cancer cells. Cancer Cell 9: 435-443, 2006.

11. Huang YW, Liu JC, Deatherage DE, et al: Epigenetic repression of microRNA-129-2 leads to overexpression of SOX4 oncogene in endometrial cancer. Cancer Res 69: 9038-9046, 2009.

12. Kulkarni S, Savan R, Qi Y, et al: Differential microRNA regulation of $H L A-C$ expression and its association with HIV control. Nature 472: 495-498, 2011.

13. Wang LH, Kim SH, Lee JH, et al: Inactivation of SMAD4 tumor suppressor gene during gastric carcinoma progression. Clin Cancer Res 13: 102-110, 2007.

14. Chern CJ and Beutler E: Biochemical and electrophoretic studies of erythrocyte pyridoxine kinase in white and black Americans. Am J Hum Genet 28: 9-17, 1976.

15. Aaboe M, Birkenkamp-Demtroder K, Wiuf C, et al: SOX4 expression in bladder carcinoma: clinical aspects and in vitro functional characterization. Cancer Res 66: 3434-3442, 2006.

16. Lee SH, Kunz J, Lin SH and Yu-Lee LY: 16-kDa prolactin inhibits endothelial cell migration by down-regulating the Ras-Tiam1Rac1-Pak1 signaling pathway. Cancer Res 67: 11045-11053, 2007.

17. van de Wetering M, Oosterwegel M, van Norren $\mathrm{K}$ and Clevers $\mathrm{H}$ : Sox-4, an Sry-like HMG box protein, is a transcriptional activator in lymphocytes. EMBO J 12: 3847-3854, 1993.

18. van Beest M, Dooijes D, van De Wetering M, et al: Sequencespecific high mobility group box factors recognize 10-12-base pair minor groove motifs. J Biol Chem 275: 27266-27273, 2000.

19. Wotton D, Lake RA, Farr CJ and Owen MJ: The high mobility group transcription factor, $\mathrm{SOX} 4$, transactivates the human $C D 2$ enhancer. J Biol Chem 270: 7515-7522, 1995.

20. Mu L, Berti L, Masserdotti G, et al: SoxC transcription factors are required for neuronal differentiation in adult hippocampal neurogenesis. J Neurosci 32: 3067-3080, 2012. 
21. Zhang J, Liang Q, Lei Y, et al: SOX4 induces epithelial-mesenchymal transition and contributes to breast cancer progression. Cancer Res 72: 4597-4608, 2012.

22. Kuwahara M, Yamashita M, Shinoda K, et al: The transcription factor Sox4 is a downstream target of signaling by the cytokine TGF- $\beta$ and suppresses $\mathrm{T}(\mathrm{H}) 2$ differentiation. Nat Immunol 13: 778-786, 2012.

23. Mavropoulos A, Devos N, Biemar F, et al: sox $4 b$ is a key player of pancreatic $\alpha$ cell differentiation in zebrafish. Dev Biol 285: 211-223, 2005.

24. Pan X, Zhao J, Zhang WN, et al: Induction of SOX4 by DNA damage is critical for $\mathrm{p} 53$ stabilization and function. Proc Natl Acad Sci USA 106: 3788-3793, 2009.

25. Ahn SG, Kim HS, Jeong SW, et al: Sox-4 is a positive regulator of Hep3B and HepG2 cells' apoptosis induced by prostaglandin (PG)A(2) and $\Delta(12)-P G J(2)$. Exp Mol Med 34: 243-249, 2002.

26. Hur EH, Hur W, Choi JY, et al: Functional identification of the pro-apoptotic effector domain in human Sox4. Biochem Biophys Res Commun 325: 59-67, 2004.

27. Mikkers H, Allen J, Knipscheer P, et al: High-throughput retroviral tagging to identify components of specific signaling pathways in cancer. Nat Genet 32: 153-159, 2002.

28. Suzuki T, Shen H, Akagi K, et al: New genes involved in cancer identified by retroviral tagging. Nat Genet 32: 166-174, 2002.

29. Ahn SG, Cho GH, Jeong SY, et al: Identification of cDNAs for Sox-4, an HMG-Box protein, and a novel human homolog of yeast splicing factor SSF-1 differentially regulated during apoptosis induced by prostaglandin $\mathrm{A}_{2} / \Delta^{12}-\mathrm{PGJ}_{2}$ in Hep3B cells. Biochem Biophys Res Commun 260: 216-221, 1999.

30. Graham JD, Hunt SM, Tran N and Clarke CL: Regulation of the expression and activity by progestins of a member of the SOX gene family of transcriptional modulators. J Mol Endocrinol 22 295-304, 1999
31. Lee CJ, Appleby VJ, Orme AT, et al: Differential expression of SOX4 and SOX11 in medulloblastoma. J Neurooncol 57: 201-214, 2002.

32. Bangur CS, Switzer A, Fan L, et al: Identification of genes over-expressed in small cell lung carcinoma using suppression subtractive hybridization and cDNA microarray expression analysis. Oncogene 21: 3814-3825, 2002.

33. Frierson HF Jr, El-Naggar AK, Welsh JB, et al: Large scale molecular analysis identifies genes with altered expression in salivary adenoid cystic carcinoma. Am J Pathol 161: 1315-1323, 2002.

34. Allegra A, Alonci A, Campo S, et al: Circulating microRNAs: New biomarkers in diagnosis, prognosis and treatment of cancer (Review). Int J Oncol 41: 1897-1912, 2012.

35. Mo MH, Chen L, Fu Y, Wang W and Fu SW: Cell-free circulating miRNA biomarkers in cancer. J Cancer 3: 432-448, 2012.

36. Garzon R and Marcucci G. Potential of microRNAs for cancer diagnostics, prognostication and therapy. Curr Opin Oncol 24: 655-659, 2012.

37. Zhao JJ, Yang J, Lin J, et al: Identification of miRNAs associated with tumorigenesis of retinoblastoma by miRNA microarray analysis. Childs Nerv Syst 25: 13-20, 2009.

38. Shen R, Pan S, Qi S, Lin X and Cheng S: Epigenetic repression of microRNA-129-2 leads to overexpression of SOX4 in gastric cancer. Biochem Biophys Res Commun 394: 1047-1052, 2010.

39. Tsai KW, Wu CW, Hu LY, et al: Epigenetic regulation of miR-34b and miR-129 expression in gastric cancer. Int J Cancer 129: 2600-2610, 2011

40. Bandres E, Agirre X, Bitarte N, et al: Epigenetic regulation of microRNA expression in colorectal cancer. Int J Cancer 125: 2737-2743, 2009. 